\title{
Use of a High-Precision Digital Displacement Encoder for Hybrid Simulation of Seismic Response of Stiff Specimens
}

Journal Article

Author(s):

Whyte, Catherine; Stojadinovic, Bozidar (1)

Publication date:

2016-04

Permanent link:

https://doi.org/10.3929/ethz-b-000476429

Rights / license:

$\underline{\text { In Copyright - Non-Commercial Use Permitted }}$

Originally published in:

Experimental Techniques 40(2), https://doi.org/10.1007/s40799-016-0069-x 


\title{
Use of a High-Precision Digital Displacement Encoder for Hybrid Simulation of Seismic Response of Stiff Specimens
}

\author{
Catherine Whyte ${ }^{1}$ and Bozidar Stojadinovic ${ }^{2}$ \\ ${ }^{1}$ Postdoctoral Researcher, Department of Civil, Environmental and Geomatic Engineering, Swiss \\ Federal Institute of Technology (ETH) Zurich (whyte@ibk.baug.ethz.ch) \\ ${ }^{2}$ Professor and Chair of Structural Dynamics and Earthquake Engineering, Department of Civil, \\ Environmental and Geomatic Engineering, Swiss Federal Institute of Technology (ETH) Zurich \\ (stojadinovic@ibk.baug.ethz.ch)
}

\begin{abstract}
Squat reinforced concrete shear walls typically comprise the lateral force resisting system in nuclear facility structures. The geometry and thickness of these walls contribute to an extremely high initial lateral stiffness. When conducting a hybrid simulation, a high stiffness presents a challenge for determining the dynamic response of such walls because very small displacements must be controlled. An extension of the hybrid simulation technique using a digital displacement encoder for high resolution displacement feedbacks was developed, implemented, verified, and validated. Then a hybrid model that represents a nuclear facility structure with a large-scale squat wall physical specimen was designed. A ground motion excitation was selected and scaled to represent specific performance levels. These preparations for hybrid simulations of the seismic response of squat shear wall specimens, subjected to different ground motion sequences, are presented to illustrate the new developments. Guidelines are provided for future implementations of feedback instruments for servo-hydraulic systems to conduct hybrid simulation tests.
\end{abstract}

Keywords: hybrid simulation; displacement control; digital displacement encoder; squat shear wall; stiff specimen

\section{INTRODUCTION}

Squat reinforced concrete shear walls commonly comprise the lateral force resisting system of heavy industrial buildings, such as nuclear power plants. These walls have a very high stiffness because of a combination of their thickness and their squat geometry. Their thickness provides radiation or contaminant shielding and blast and fire resistance, and their aspect ratio, driven by the industrial technology requirements, is commonly less than 0.5 . There is a substantial gap in our understanding of the behavior of these types of walls as exemplified by the poor ability of structural design code equations to predict wall peak shear strengths and deformations. Gulec's ${ }^{1}$ database of squat shear wall tests demonstrates a large scatter in the ratio of predicted shear strength and deformation by design code equations to corresponding measured values. Most previous squat wall tests have been conducted with small-scale models exposed to standard quasi-static cyclic load sequences. ${ }^{2}$ There are two concerns with this approach: 1) the size effect presents a significant obstacle to interpreting small-scale model test results; and 2) it is unclear whether a quasi-static cyclic test is adequate to capture the complex interaction of failure modes that characterize the seismic response of squat shear walls. 
To address these concerns, a series of large-scale tests, conducted with the goal of improving the understanding of squat wall behavior in response to earthquake ground motion excitations, was recently completed with a joint National Science Foundation (NSF) NEES-R project. $^{3,4}$ Following consultations with practicing engineers, the prototype was selected as a squat (aspect ratio of 0.5 ), $90 \mathrm{~cm}$ (36 in) thick reinforced concrete shear wall with minimum reinforcement, typical for a nuclear facility structure. Three nominally identical model shear walls were constructed with $20.3 \mathrm{~cm}$ (8 in) thickness and an aspect ratio of 0.53 . One wall was tested at the University at Buffalo nees@Buffalo Equipment Site (http://nees.buffalo.edu) using a quasi-static testing protocol with an incrementally increasing horizontal displacement sequence. ${ }^{4}$ Two specimens were tested at the University of California, Berkley nees@Berkeley Equipment Site (http://nees.berkeley.edu) using the hybrid simulation method to simulate the seismic response of a prototype nuclear facility structure, including the model shear wall, to different ground motion excitation sequences. Details about hybrid simulation results and the comparison between the quasi-static and the hybrid simulation tests are provided by Whyte and Stojadinovic. ${ }^{5}$ The focus of this paper is the extension of the hybrid simulation method ${ }^{6}$ to attain adequate performance for response simulation using hybrid models with very stiff and strong specimens. Details are presented about the servo-hydraulic control feedback loop that was developed and implemented for this purpose, about the definition of the hybrid model, and about the selection of ground motion scaling factors to model an operational basis earthquake (OBE), a design basis earthquake (DBE), and a beyond design basis earthquake (BDBE) ground motion for the hybrid response simulation sequences.

\section{HYBRID SIMULATION AND APPLICATION TO STIFF SPECIMENS}

Hybrid simulation is an experimentally based method for investigating the dynamic response of structures using a hybrid model. The hybrid model comprises physical and numerical substructures, i.e. most of the prototype, including the loads, is modeled with finite element software while the portion of the prototype, whose response is difficult to model numerically, is tested physically in a structural testing laboratory. The force equilibrium and displacement continuity at the interface between the physical and numerical substructures are maintained using servo-hydraulic jacks and closed-loop controllers. The dynamic response of the hybrid model is obtained using a time-stepping integration method that solves the equation of motion describing the entire hybrid model. In a displacement control approach, the resulting displacements of the experiment element(s) are applied to the physical specimen at each time step. Load cells measure the restoring forces of the physical specimen, and this information is returned to the hybrid model for calculating the motion in the next time step.

Hybrid simulation with stiff and strong physical specimens, such as the squat shear walls, poses a number of challenges. Large force increments, possible if the simulations are conducted in displacement control, must be avoided for the following reasons. (1) They could cause an abrupt change in the specimen failure mode, and worse, induce a sudden quasi-brittle failure. This could lead to erroneous conclusions because the change in specimen behavior would be the result of a poorly-controlled simulation rather than the actual effect of dynamic loads. (2) Large force increments could destabilize the numerical portion of the hybrid model or cause a convergence failure of the simulation time-stepping integrator. The displacement increment required to achieve a sufficiently small force increment may become so small that it approaches the resolution of the displacement feedback measurement and the tolerance of the actuator 
control system. Alternatively, conduct of the hybrid simulation in force control is possible, but it requires good estimates of the specimen's initial and subsequent tangent stiffnesses, which are also obtained from accurate measurements of small changes in displacement.

\section{Hybrid Simulation at the nees@Berkeley Equipment Site}

For the hybrid simulation tests of the seismic response of squat shear walls, the Open System for Earthquake Engineering Simulation (OpenSees) ${ }^{7}$ Framework was used to model the numerical portion of the hybrid model. The Open-source Framework for Experimental Setup and Control (OpenFresco $)^{8}$ was used to represent the physical wall specimen as an experimental element in the hybrid model, transform between degrees-of-freedom (DOFs) in the hybrid model and the actuator DOFs in the laboratory, and perform the experimental control. OpenFresco is a flexible client-server framework, suitable for incorporating various finite element software and laboratory hardware. ${ }^{9}$ Communication between OpenFresco and the digital MTS Structural Test Systems (STS) Controller ${ }^{10}$, which links to the servo-valves, actuators, and laboratory specimens, was realized using an XPC Target experimental control.

The xPC Target experimental control uses an xPC host computer and an xPC target realtime processor, and communicates with the STS Controller. OpenSees and OpenFresco run on the $\mathrm{xPC}$ host computer, and a Simulink ${ }^{11}$ predictor-corrector $(\mathrm{P} / \mathrm{C})$ model runs on the $\mathrm{xPC}$ target computer. Target displacements generated in the finite element model are sent from the $\mathrm{xPC}$ host to the $\mathrm{P} / \mathrm{C}$ model on the $\mathrm{xPC}$ target computer over a TCP/IP connection. This $\mathrm{P} / \mathrm{C}$ model extrapolates past target displacements and sends substep command displacements to the STS Controller at the required $1024 \mathrm{~Hz}$ rate via a shared memory real-time communications network, SCRAMNet $^{12}$, which updates its memory at the same $1024 \mathrm{~Hz}$ rate. A D3D3 P/C model, provided with the OpenFresco software package, is based on extrapolation and interpolation of the three prior displacements using third-order Lagrange polynomials during prediction and correction phases. ${ }^{13}$ While this $\mathrm{P} / \mathrm{C}$ model waits for a new target displacement from the finite element model (which arrives at a much slower and less reliable rate than $1024 \mathrm{~Hz}$ ), it sends predictions of the next command displacements to the STS Controller at the $1024 \mathrm{~Hz}$ rate. When the $\mathrm{P} / \mathrm{C}$ model receives the new target displacement from the finite element model, it sends corrections to the STS Controller, so it smoothly transitions to that next target displacement value.

\section{Equipment Considerations}

The hybrid simulation setup at the nees@Berkeley Equipment Site used an analog linear variable differential transformer (LVDT) to measures displacements in the actuator servohydraulic control system. A typical LVDT feedback setup can control displacement increments as small as $0.025 \mathrm{~mm}$ (0.001 in). By using a shorter stroke, a LVDT can achieve an even better resolution. The concern with the squat wall tests was the maximum displacements of the walls were unknown before the tests. Though the walls were very stiff, they ultimately failed in sliding shear, so potentially large sliding displacements would need to be measured. Thus, the authors did not want to limit the range of the LVDT transducer. Instead, a Heidenhain LS 623 digital displacement encoder (DDE) with a stroke of $1140 \mathrm{~mm}$ and a step resolution of $0.01 \mathrm{~mm}(0.0004$ in) was implemented to feed back displacements in the actuator control loop. The advantage of the DDE is that it maintains this (very high) resolution over the entire length of the instrument 
stroke. Such improvement in displacement control resolution was expected to keep the force increments at acceptably small levels during the hybrid simulations while being able to capture the sliding motions of the walls without any restriction in the sliding magnitude.

A $6670 \mathrm{kN}$ (1500 kips) servo-hydraulic actuator was used for the squat wall tests. The actuator was known to have small gaps in its clevis connections. In a previous pseudo-dynamic test of a steel braced frame using the same actuator, hybrid simulation results were improved by moving the specimen using a constant-velocity command signal. The notion of time in the hybrid model was unchanged, but the number of substeps sent to the STS Controller (at the $1024 \mathrm{~Hz}$ rate) was adjusted on the fly so the actuator moved continuously at a set velocity. ${ }^{14}$ Though larger displacements took a greater amount of time to apply to the physical specimen, this was not believed to cause any additional errors because the test was already being performed pseudodynamically at a very slow rate. The constant velocity command signal strategy limited the displacement control errors to the times when the actuator changed directions, and the gaps in the clevises either opened or closed. The same strategy was adopted for the squat wall tests. Pretests with the actuator, described below, were conducted using constant velocity triangle wave motions.

The D3D3 P/C model with the constant velocity modification was used for the braced frame tests ${ }^{14}$. This model was modified and named E3E3 (for encoder) for use with the DDE for the hybrid simulations of the response of the squat shear wall specimens. The size of the User Datagram Protocol (UDP) block in the E3E3 P/C model was increased to include new DDE commands and feedbacks. The model handles the DDE commands and feedbacks in the same manner as the LVDT commands and feedbacks were handled in the original $\mathrm{P} / \mathrm{C}$ model. For the E3E3 model to communicate with the STS controller, DDE commands and feedbacks had to be accessible over specifically designated channels in the SCRAMNet shared memory network map. This map is tailored to the particular nees@Berkeley Equipment Site hybrid simulation setup, and MTS Systems Corp. added channels to the SCRAMNet memory map for the DDE input from SCRAMNet and the DDE output to SCRAMNet.

The Moog 72-103 servovalve connected to the actuator, which was rated at $227 \mathrm{~L} / \mathrm{min}$ (60 gal $/ \mathrm{min}$ ), was underperforming considerably. The flow at peak velocity was computed to be about 30 times less than the rated flow with no load. The cable connected to the servovalve was initially wired in parallel, which supplies each coil of the servovalve with half of the applied current. The cable was rewired in series to give the same current to both coils, resulting in twice the supply of current to the servovalve. This was expected to yield twice better performance, which was confirmed by testing both wiring configurations, described below.

\section{DDE Verification and Validation without a Specimen}

The performance of the DDE was first compared to the performance of a LVDT by monitoring actuator displacements directly. The actuator was disconnected from the strong wall and placed on the laboratory floor, such that it had no resistance to its motion. Small-magnitude triangle wave displacement signals were commanded to the actuator, and both the LVDT and the DDE measured the motion of the actuator clevis relative to the actuator body. The test setup is shown in Figure 1. First, the LVDT was used to provide displacement feedbacks for the purpose 
of controlling the actuator, and the DDE displacements were recorded. Then the DDE was used to provide displacement feedbacks, and the LVDT displacements were recorded.

In order to test the actuator performance with the different servovalve wiring configurations, a triangle wave displacement motion with a $25.4 \mathrm{~mm}$ (1 in) amplitude was commanded for the following three cases: LVDT control with wiring in parallel, LVDT control with wiring in series, and DDE control with wiring in series. The comparisons of errors between command and feedback displacements for the three cases are shown for velocities of $2.032 \mathrm{~mm} / \mathrm{s}$ $(0.08 \mathrm{in} / \mathrm{s})$ and $6.096 \mathrm{~mm} / \mathrm{s}(0.24 \mathrm{in} / \mathrm{s})$ in Figures 2 and 3, respectively. It is evident from these plots that the performance of the DDE with the wiring in series is superior to that of the LVDT for minimizing displacement errors between command and feedback displacements. This series wiring was retained for all future tests. It is also evident that the error plots contain spikes but only when the actuator changes its direction of motion and the clevis gaps open or close, as expected. The normalized percent errors for each of these three configurations at both velocities tested are presented in Table 1. The normalized percent error was calculated by dividing the peak displacement error by its respective commanded displacement, expressed as a percentage. The table shows the overall peak values, considering all time steps for each configuration. This includes the error spikes when the actuator changed directions. The normalized percent errors considering just the portions of the plots when the actuator was moving at constant velocity are also displayed. The lower part of the table shows the DDE Series percent improvement over the LVDT Series and the LVDT Parallel configurations. At constant velocity, the DDE Series configuration improved performance by $54.4 \%$ compared to the best LVDT Series configuration at $2.032 \mathrm{~mm} / \mathrm{s}$ and by $27.1 \%$ at $6.096 \mathrm{~mm} / \mathrm{s}$.

For the Proportional, Integral, Derivative (PID) feedback loop, only a large Proportional gain of 200 was used, while the Integral and Derivative gains were set to 0 . These gains were maintained for both the parallel and series wiring configurations, as well as for all future tests.

\section{DDE Verification and Validation using a Braced Frame Specimen}

After validating the DDE performance using an unrestrained actuator, its performance in the control loop was tested in three configurations using a braced frame specimen from a previous experiment ${ }^{14}$ to provide resistance to actuator motion. The three configurations are summarized in Table 2. In the first configuration, the actuator was mounted to the strong wall and connected to the braced frame specimen. As done previously, both the LVDT and the DDE measured the motion of the actuator clevis, in turn, relative to the actuator body. A triangle wave displacement motion with a $7.62 \mathrm{~mm}$ (0.3 in) amplitude was commanded at a velocity of 0.381 $\mathrm{mm} / \mathrm{s}(0.015 \mathrm{in} / \mathrm{s})$. The comparison of errors between displacement commands and feedbacks recorded during LVDT control and during DDE control are shown in Figure 4. The LVDT errors remained within about $0.2 \mathrm{~mm}(0.0079 \mathrm{in})$ while the DDE errors remained within about $0.12 \mathrm{~mm}$ (0.0047 in) when the actuator changed directions. Otherwise, the LVDT errors were within about $0.15 \mathrm{~mm}(0.0059 \mathrm{in})$ while the DDE errors were within about $0.05 \mathrm{~mm}(0.0020 \mathrm{in})$. At constant velocity, the LVDT errors were approximately six times higher and the DDE errors were approximately five times higher than their respective nominal resolutions. The normalized percent errors are presented in Table 3. At constant velocity, the DDE improved performance by $67.5 \%$ compared to the LVDT. 
In the second configuration, shown in Figure 5a, a stiff reference frame was installed next to, but separate from, the braced frame specimen. The DDE was mounted on this frame and fed back the displacements of the top of the first story column of the braced frame via a long pole (the label, "Attachment," shows the connection point to the braced frame's column). Again, a triangle wave displacement motion with a $7.62 \mathrm{~mm}(0.3 \mathrm{in})$ amplitude was commanded at a velocity of $0.381 \mathrm{~mm} / \mathrm{s}(0.015 \mathrm{in} / \mathrm{s})$, producing similar results.

In the third configuration, the DDE measured the relative displacement between the reference frame and the beam at the top of the first story of the braced frame via a connection designed for use in the hybrid simulation tests of the response of the squat shear wall specimens, shown in Figure 5b. This is a view from above of the location labeled "DDE" in Figure 5a. A flexible steel plate connected the DDE to the specimen. This allowed for vertical motion but restrained horizontal motion. A linear guide block accommodated any out-of-plane movements by sliding along a rail, and a 360 degree swivel accommodated any torsion of the specimen. For just this pretest with the braced frame specimen (not for the future squat shear wall specimen implementation), the baseplate of the linear guide block was attached to a small I-beam, which was clamped to the top of the braced frame's beam. This was done because the reference frame was taller than the braced frame's beam, and the DDE needed a reference point at the same height. A triangle wave displacement motion with a $7.62 \mathrm{~mm}(0.3 \mathrm{in})$ amplitude was commanded at a velocity of $0.381 \mathrm{~mm} / \mathrm{s}(0.015 \mathrm{in} / \mathrm{s})$ for this configuration too.

The errors from the DDE referencing the actuator, column, or beam are shown in Figure 6: they remained within about $0.12 \mathrm{~mm}(0.0047 \mathrm{in})$ when the actuator changed directions. Otherwise, the error was generally within $0.05 \mathrm{~mm}(0.0020 \mathrm{in})$. If the actuator clevises had been machined better, the displacement control fidelity using the DDE would have been improved. The normalized percent errors are presented in Table 4. At constant velocity, the resulting errors were $0.86 \%$ for the DDE referencing the actuator, $1.09 \%$ for the DDE referencing the column, and $1.14 \%$ for the DDE referencing the beam. The three configurations show approximately the same performance with overall normalized errors less than $1.5 \%$.

After the modifications made to the hybrid simulation software to include the DDE were validated and verified, the final test with the braced frame specimen was a hybrid simulation of the seismic response of the frame to the 1978 Tabas Earthquake ground motion, scaled to $10 \%$ of its recorded intensity for the first 500 time steps. This motion was commanded at a $0.381 \mathrm{~mm} / \mathrm{s}$ $(0.015 \mathrm{in} / \mathrm{s})$ constant velocity using an implicit integration method. The commanded displacements, feedback displacements, and errors from the DDE control are shown in Figure 7, as "Cmd," "Fbk," and "Err," respectively. For approximately the first 25 seconds, the errors are approximately the same magnitude as the command signal because it was difficult to control such small displacements given the problems with the actuator clevises. When the actuator changes directions, it needs to travel a certain distance for the clevis gaps to either open or close. For very small displacement commands, the clevises might be opening or closing for nearly the entire commanded displacement. Disregarding the first 25 seconds in Figure 7, the error was approximately $0.18 \mathrm{~mm}$ (0.007 in) overall (4.50\% normalized error) and $0.078 \mathrm{~mm}(0.003 \mathrm{in})$ during constant velocity portions $(2.26 \%$ normalized error). This test confirmed satisfactory behavior of the hybrid simulation test setup with the DDE providing displacement feedback. 


\section{HYBRID SIMULATION SETUP AND EXECUTION}

After the performance of the DDE as a displacement feedback device was validated and verified and a hybrid simulation test using the DDE with the braced frame was successful, the test setup was ready to accommodate the first squat reinforced concrete shear wall specimen, as shown in Figure 8. The stiff reference frame and encoder were relocated to the back of the specimen. Additional work was necessary to define the hybrid model and select and scale the ground motions.

\section{Hybrid Model and Pretests}

The hybrid model for the prototype nuclear facility structure consisted of a squat shear wall physical specimen and a large mass modeled numerically using OpenSees, such that the undamaged natural period of vibration of the hybrid model matched the $0.14 \mathrm{~s}$ fundamental natural vibration period of a typical nuclear structure. This period value was based on a model of a Candu Reactor created by Huang et al. ${ }^{15,16}$ The experimental wall element was fixed at its base and had a single horizontal degree of freedom where the actuator attaches to the wall, as shown in Figure 8. Prior to performing the hybrid simulation test using a ground motion excitation, the first squat shear wall specimen was installed and subjected to a small-amplitude displacement pretest and a free vibration pretest.

The small-amplitude displacement test was used to measure the initial stiffness of the specimen and to determine the required mass for the numerical model to achieve the $0.14 \mathrm{~s}$ fundamental vibration period. This test consisted of a small triangle wave displacementcontrolled motion applied using forces limited to $+/-444.8 \mathrm{kN}$ (+/-100 kips). Figure 9 shows the displacement commands and feedbacks vs time, force vs time, and hysteretic responses for the first wall's stiffness test. The displacement feedbacks did not track the displacement commands well when the actuator changed directions at very small displacements due to the gaps in the clevises of the actuator. Thus, the force response was also not very smooth. The stiffness was estimated to be $840.6 \mathrm{kN} / \mathrm{mm}(4800 \mathrm{k} / \mathrm{in})$, and thus the mass implemented in the hybrid model was $0.417 \mathrm{kN}-\mathrm{s}^{2} / \mathrm{mm}\left(2.383 \mathrm{kip}-\mathrm{s}^{2} / \mathrm{in}\right)$.

In the free vibration test, the hybrid model was first driven to a starting displacement of $0.224 \mathrm{~mm}(0.0088 \mathrm{in})$ at the same $2.54 \mathrm{~cm} / \mathrm{s}(0.01 \mathrm{in} / \mathrm{s})$ constant velocity intended to be used later for the hybrid simulation tests. This displacement value kept the actuator forces within $+/-$ $222.4 \mathrm{kN}$ (+/-50 kips). Then the hybrid model was released to vibrate freely. The free vibration displacements are shown in Figure 10. The damping in this free vibration was assessed using the decrement method and found to be minimal. Therefore, mass-proportional damping equal to $2 \%$ of critical was implemented directly in the numerical portion of the hybrid model.

The fidelity of the DDE can be assessed by its adequacy for performing the free vibration test. With a measured maximum normalized error of $1.14 \%$ at constant velocity, the error to travel to the $0.224 \mathrm{~mm}(0.0088 \mathrm{in})$ free vibration test starting displacement is $0.0031 \mathrm{~mm}(0.001$ in). This gives an error resolution of about $1 / 72$, which is sufficiently small to capture the free vibration response. The force error, which is the product of the displacement error and the specimen stiffness, is $26.1 \mathrm{kN}$ (5.868 kips). 


\section{Ground Motion Selection and Scaling}

The nuclear facility prototype was considered to be located at the Diablo Canyon Nuclear Power Plant in San Luis Obispo County, CA, which is a Western US rock site. Huang and Whittaker ${ }^{15}$ matched 30 ground motions to the DBE spectrum for this site over a wide range of periods using seed motions from the PEER NGA Database. ${ }^{17}$ Since the fundamental period of the hybrid model was set at $0.14 \mathrm{~s}$, but was expected to elongate as the shear wall specimen experienced damage during the hybrid simulations with increasing ground motion intensity, the ground motion that best matched the DBE spectrum in the $0.14 \mathrm{~s}$ to $0.3 \mathrm{~s}$ period range was selected from Huang and Whittaker's 30 motion set. This motion was recorded during the 1999 Kocaeli, Turkey earthquake. In order to design sequences of ground motions for the two hybrid simulations, the Kocaeli motion was used as a base motion and further scaled to levels that produce behaviors expected in an OBE, DBE, or BDBE in a nuclear facility structure. Since these structures are designed to remain "essentially elastic" in a DBE event, they should remain elastic in an OBE and develop some inelastic response in a BDBE. Thus, the targeted force level for the $\mathrm{OBE}$ was $1 / 3$ of the specimen yield force, consistent with nuclear facility design goals. The targeted force level for the DBE was $2 / 3$ of the specimen yield force. The BDBE was scaled to represent an extremely large event to push the wall beyond its peak shear strength, but not to cause extreme displacements. In order to determine the appropriate scaling factors for the Kocaeli base ground motion, a simple bilinear single-degree-of-freedom (SDOF) oscillator model was created in OpenSees. A quasi-static cyclic test of a nominally identical squat shear wall specimen had been completed at the State University at Buffalo, New York, ${ }^{4}$ so the 1500 $\mathrm{kN}$ (337.2 kips) yield force observed in that test was used in the SDOF oscillator material model. The measured initial stiffness of the first shear wall specimen, the calculated mass corresponding to the $0.14 \mathrm{~s}$ fundamental vibration period, and the $2 \%$ damping were included. A trial and error response analysis using the OpenSees SDOF model was then used to determine scaling factors for the Kocaeli motion for the OBE, DBE and BDBE ground motion levels.

The following ground motion sequence was selected for the first squat shear wall specimen test: OBE, DBE, BDBE, and then a DBE aftershock. The integration time step for the hybrid model was chosen to be $0.005 \mathrm{~s}$, which was half of the Kocaeli ground motion $0.01 \mathrm{~s}$ recorded time step. The hybrid model was solved using an Explicit Newmark integrator. Then the displacements were applied to the physical wall specimen at the $2.54 \mathrm{~cm} / \mathrm{s}(0.01 \mathrm{in} / \mathrm{s})$ constant velocity, mentioned previously. The displacement and force time histories for the first 15 seconds of the OBE motion are shown in Figure 11. The force response was much improved with these slightly larger displacements than observed in the stiffness test initially. After the DBE motion was completed, the resulting peak displacement was found to be larger and the resulting peak force was found to be smaller than the target values from the SDOF model. In the SDOF model, the specimen was assumed to retain the same stiffness following the OBE motion since the displacements and forces were still very small, but the real wall specimen was found to be losing stiffness rapidly. The FFT of the recorded acceleration from the OBE motion revealed that the stiffness had dropped to $648.5 \mathrm{kN} / \mathrm{mm}(3703 \mathrm{k} / \mathrm{in})$ by the end of that motion, which was a loss of approximately $25 \%$ compared to the initially measured stiffness. The OpenSees SDOF model was updated with the new stiffness to attempt to replicate the observed behavior, but this was unsuccessful. This model was not sophisticated enough to capture the behavior of the wall including cracking, so a trilinear material model may have been more suitable. Because the DBE motion resulted in a force that was smaller than the target force, the motion was repeated with a 
larger scaling factor. This was done by linearly scaling the ground motion factor by the ratio of the desired to observed force, and this new scaling factor was retained for all future DBE motions. After repeating the DBE motion, the scaling factor for the BDBE was modified to be 3 times the DBE motion. The squat shear wall specimen had not sustained any significant damage following the DBE motion, so a large enough motion was needed to push it beyond its peak strength. Then the DBE aftershock used the same scaling factor as the DBE. A summary of the ground motion scaling factors is included in Table 5. The same scaling factors were used for the second squat shear wall specimen test, which experienced the following ground motion sequence: OBE, BDBE, DBE aftershock 1, and then DBE aftershock 2.

At the end of both ground motion simulation sequences, the specimens had lost most of their strength, and conducting further ground motion tests was determined to be a laboratory safety hazard. Instead, the specimens were subjected to quasi-static single-cyclic triangle wave motions to $+/-2.54 \mathrm{~cm}(+/-1$ in) and then to $+/-3.81 \mathrm{~cm}(+/-1.5 \mathrm{in})$ peak displacements until failure. Details about the results from these hybrid simulation tests and comparisons to the test at Buffalo can be found in Whyte and Stojadinovic. ${ }^{5}$

This process of attempting to scale the ground motions using a SDOF model illustrates the fact that the behavior of the hybrid model is difficult to capture numerically beyond the smallest motion magnitudes while the specimen remains essentially elastic and virtually uncracked. The rapid degradation of specimen stiffness must be accounted for by developing a more sophisticated numerical model for the response range below the peak resistance of the specimen. On the other hand, to model the response beyond the peak strength, maximum displacement and ductility estimates must be made while accounting for the still-substantial remaining stiffness and the relatively short fundamental vibration period of the hybrid model.

\section{CONCLUSIONS}

Nuclear facility squat reinforced concrete shear walls are very stiff and strong structural elements. Conventional displacement control in hybrid simulation is not adequate because displacement feedback increments are not small enough to keep force increments below acceptable levels for such stiff specimens without compromising the range of the displacement feedback instrument. In response to this problem, a high-precision displacement control method using a DDE for displacement feedbacks was developed at the nees@Berkeley Equipment Site. This paper described the validation and verification of an extension of the hybrid simulation seismic response simulation method to include a high-precision displacement feedback instrument in the servo-hydraulic control loop. The process for creating the hybrid model and for selecting and scaling the ground motion excitation to achieve a graded incrementally increasing intensity of stiff specimen response was also described. The difficulty of appropriately scaling the ground motion using a simple numerical model was highlighted. It is very difficult to capture the interaction of the shear, flexure, and sliding shear failure modes observed, with a simple numerical model.

The resolution, accuracy, and precision of the DDE are important considerations for acceptable performance of the servo-hydraulic actuation system. The step resolution of the DDE was $0.01 \mathrm{~mm}(0.0004 \mathrm{in})$, which was finer than the approximately $0.025 \mathrm{~mm}(0.001 \mathrm{in})$ resolution of a typical LVDT feedback setup. The DDE proved to be more accurate than the 
LVDT because it returned smaller errors between command and feedback displacements. The DDE also proved to be more precise than the LVDT because it is a digital instead of analog instrument and consistently produced less dispersion in consecutive measurements of the same displacement. The acceptance criterion for the DDE is based on limiting the size of the force increments. Larges changes in force could accidentally induce or prevent a brittle failure or cause a numerical instability in the hybrid model. Furthermore, small force increments were necessary to conduct a free vibration test to measure the damping of the specimen. With improved actuator performance, the free vibration test could have been conducted using force control. For this application, considering the gaps in the clevises of the actuator, displacement control was safer.

Based on the experience gained, the authors propose a procedure for selection, validation and verification of feedback instruments to be used in servo-hydraulic systems to conduct hybrid simulation tests. The procedure is based on defining acceptable performance parameters of the servo-hydraulic system. These parameters should be derived from the minimum force or deformation limits form the required pre-tests, from the maximum tolerable errors required for stable time-history integration of the hybrid simulation model, and maximum tolerable force or deformation increments required to accurately interpret the response of the physical specimen and/or the hybrid model. Once determined, these acceptable performance criteria can be translated into the required accuracy, precision, and resolution requirements for the feedback instruments. A verification procedure should be used to demonstrate that the stated resolution requirements have been achieved. This verification procedure comprises driving an unrestrained and a restrained servo-hydraulic system using a saw-tooth command signal centered at zero feedback level at several different constant velocities. This procedure has been implemented for a single-actuator hybrid setup.

This extension to the hybrid simulation method is important because it enables accurate simulation of the dynamic response of structures with very stiff elements, particularly when the expected response of such elements is quasi-brittle. In future efforts, displacement control precision will be further improved through the use of even more precise displacement feedback instruments, mechanical slip will be eliminated by re-machining the actuator clevises, and servovalve performance will be further enhanced to perform faster hybrid simulation tests. Additionally, a multi-actuator validation and verification procedure should be developed.

One promising direction for further extension of the hybrid simulation method is the implementation of force control methods for stiff specimens using acceleration-based feedback. ${ }^{18}$ Another is the extension from one to multiple degrees-of-freedom, some controlled in force, others in displacement, with the stiffnesses of the specimen varying significantly depending on the direction of loading. An example of such mixed control hybrid simulation would involve the same squat reinforced concrete wall specimens carrying a substantial gravity load, simulated by applying constant vertical force on the specimens.

\section{ACKNOWLEDGMENTS}

The authors are grateful to Prof. Andrew Whittaker and Mr. Bismarck Luna at the State University of New York at Buffalo and Prof. Laura Lowes at the University of Washington for their contributions and discussions of test data. Funding for this project was provided by NSF 
NEES-R grant CMMI-0829978. Any opinions, findings, and conclusions expressed herein are those of the author and do not necessarily reflect the view of the National Science Foundation.

\section{REFERENCES}

[1] Gulec, C. K., and Whittaker, A. S. "Performance-Based Assessment and Design of Squat Reinforced Concrete Shear Walls", Technical Report MCEER-09-0010, University at Buffalo, State University of New York at Buffalo, Buffalo, NY, 2009.

[2] Leon, R. T. and Deierlein, G. G. "Considerations for Use of Quasi-Static Testing”, Earthquake Spectra, Vol. 12, No. 1, pp. 87-109, 1996.

[3] Whyte, C. A. and Stojadinovic, B. "Hybrid Simulation of the Seismic Response of Squat Reinforced Concrete Shear Walls", PEER Center Research Report No. 2013/02, May 2013, pp. 1-196, 2013.

[4] Rocks, J. F., Luna, B. N. and Whittaker, A. S. “Seismic Response of Low Aspect Ratio Reinforced Concrete Shear Walls", Proc., SMiRT 21, New Delhi, India, 2011.

[5] Whyte, C. A. and Stojadinovic, B. "Effect of ground motion sequence on the response of squat reinforced concrete shear walls", Journal of Structural Engineering, doi:10.1061/(ASCE)ST.1943-541X.0000912, 2013.

[6] Saouma V. E., and Sivaselvan, M. V. "Hybrid Simulation: Theory, Implementation and Applications", Taylor and Francis, London, UK, 2008.

[7] OpenSees. Open System for Earthquake Engineering Simulation.

http://opensees.berkeley.edu, 2013.

[8] OpenFresco. Open Framework for Experimental Setup and Control.

http://openfresco.neesforge.nees.org, 2013.

[9] Schellenberg, A. and Mahin, S. "Application of an Experimental Software Framework to Hybrid Simulation of Structures through Collapse", $1^{\text {st }}$ European Conference on Earthquake Engineering and Seismology, Geneva, Switzerland, 2006.

[10] MTS. Model 793.00 System Software, User Information and Software Reference, MTS Systems Corporation, 2003.

[11] Mathworks. MATLAB, Simulink, Stateflow, xPC Target, http://www.mathworks.com, 2012.

[12] Systran. SCRAMNet+ Network, http://www.cwcdefense.com/products/io-communicationboards/, 2000.

[13] Schellenberg, A. “Advanced Implementation of Hybrid Simulation”, Ph.D. Thesis, University of California, Berkeley, 2008.

[14] Lai, J. W. "Experimental and Analytical Studies on the Seismic Behavior of Conventional and Hybrid Braced Frames", Dissertation, Department of Civil and Environmental Engineering, University of California, Berkeley, Berkeley, CA, 2012.

[15] Huang, Y. N. and Whittaker, A. S. "Performance Assessment of Conventional and BaseIsolated Nuclear Power Plants for Earthquake and Blast Loadings", Technical Report MCEER08-0019, State University of New York at Buffalo, Buffalo, NY, 2008.

[16] Huang, Y. N. et al. "Assessment of Base-Isolated Nuclear Structures for Design and Beyond-Design Basis Earthquake Shaking”, Technical Report MCEER-09-0008, State University of New York at Buffalo, Buffalo, NY, 2009.

[17] PEER NGA Database. http://peer.berkeley.edu/peer_ground motion_database, 2012. 
[18] Nakata, N. and Krug, E. "Multi-Degrees-of-Freedom Effective Force Testing: A Feasibility Study and Robust Stability Assessment", Earthquake Engineering and Structural Dynamics, DOI: 10.1002/eqe.2308, 2013. 
Table 1. Normalized Percent Error for Three Configurations (Figures 2 and 3)

\begin{tabular}{c|c|c|c|c} 
& \multicolumn{2}{|c|}{ Overall Peak Values } & \multicolumn{2}{c}{ At Constant Velocity } \\
\hline Configuration & $\mathrm{V}=2.032 \mathrm{~mm} / \mathrm{s}$ & $\mathrm{V}=6.096 \mathrm{~mm} / \mathrm{s}$ & $\mathrm{V}=2.032 \mathrm{~mm} / \mathrm{s}$ & $\mathrm{V}=6.096 \mathrm{~mm} / \mathrm{s}$ \\
\hline LVDT Parallel & 2.11 & 5.13 & 1.50 & 3.77 \\
\hline LVDT Series & 1.35 & 2.91 & 1.14 & 2.66 \\
\hline DDE Series & 1.09 & 2.41 & 0.52 & 1.94 \\
\hline
\end{tabular}

\begin{tabular}{c|c|c|c|c} 
& \multicolumn{4}{|c}{ DDE Percent Improvement } \\
\hline $\begin{array}{c}\text { DDE Series/ } \\
\text { LVDT Series }\end{array}$ & 19.3 & 17.2 & 54.4 & 27.1 \\
\hline $\begin{array}{c}\text { DDE Series/ } \\
\text { LVDT Parallel }\end{array}$ & 48.3 & 53.0 & 65.3 & 48.5
\end{tabular}


Table 2. Summary of DDE configurations tested using the braced frame specimen

\begin{tabular}{c|c|c} 
Configuration & DDE Position & Measured Displacements \\
\hline 1 & Actuator body & Actuator clevis relative to actuator body \\
\hline 2 & Reference frame & $\begin{array}{c}\text { Braced frame column relative to reference frame } \\
\text { via pole attachment }\end{array}$ \\
\hline 3 & Reference frame & $\begin{array}{c}\text { Braced frame first story beam relative to reference } \\
\text { frame via plate/guide block/swivel connection }\end{array}$
\end{tabular}


Table 3. Normalized Percent Error for LVDT and DDE (Figure 4)

\begin{tabular}{c|c|c} 
Configuration & Overall Peak Values & At Constant Velocity \\
\hline LVDT & 2.55 & 2.40 \\
\hline DDE & 1.50 & 0.78 \\
\hline
\end{tabular}

\begin{tabular}{l|c|r} 
& \multicolumn{2}{|c}{ DDE Percent Improvement } \\
\hline DDE / LVDT & 41.2 & 67.5 \\
\hline
\end{tabular}


Table 4. Normalized Percent Error for Three Configurations (Figure 6)

\begin{tabular}{c|c|c} 
Configuration & Overall Peak Values & At Constant Velocity \\
\hline LVDT Parallel & 1.50 & 0.86 \\
\hline LVDT Series & 1.01 & 1.09 \\
\hline DDE Series & 1.47 & 1.14 \\
\hline
\end{tabular}


Table 5. 1999 Kocaeli Earthquake ground motion scaling factors

\begin{tabular}{c|c|c} 
GM Level & PGA $(\mathrm{g})$ & Scaling Factor \\
\hline OBE & 0.043 & 0.053 \\
\hline DBE & 0.12 & 0.14 \\
\hline BDBE & 0.35 & 0.42
\end{tabular}




\section{LIST OF FIGURES}

Figure 1: DDE verification using the servo-hydraulic actuator without a specimen.

Figure 2: Displacement errors for LVDT parallel, LVDT series, and DDE series wiring using the unrestrained servo-hydraulic actuator at velocity $=2.032 \mathrm{~mm} / \mathrm{s}$.

Figure 3: Displacement errors for LVDT parallel, LVDT series, and DDE series wiring using the unrestrained servo-hydraulic actuator at velocity $=6.096 \mathrm{~mm} / \mathrm{s}$.

Figure 4: Displacement errors for LVDT and DDE servo-hydraulic actuator control using the braced frame specimen.

Figure 5a: Braced frame specimen, reused for DDE validation.

Figure 5b: DDE connection designed for hybrid simulations of the response of squat shear wall specimens; here, tested using the braced frame specimen.

Figure 6: Displacement errors for DDE control using actuator, column, or beam reference points with the braced frame specimen.

Figure 7: Displacement commands, feedbacks, and errors for hybrid simulation of the response of the braced frame specimen to $10 \%$ of the 1978 Tabas Earthquake ground motion.

Figure 8: Global view of the squat reinforced concrete shear wall specimen test setup.

Figure 9: Wall 1 stiffness test results.

Figure 10: Wall 1 free vibration test results.

Figure 11: Wall $1 \mathrm{OBE}$ displacement and force time histories. 


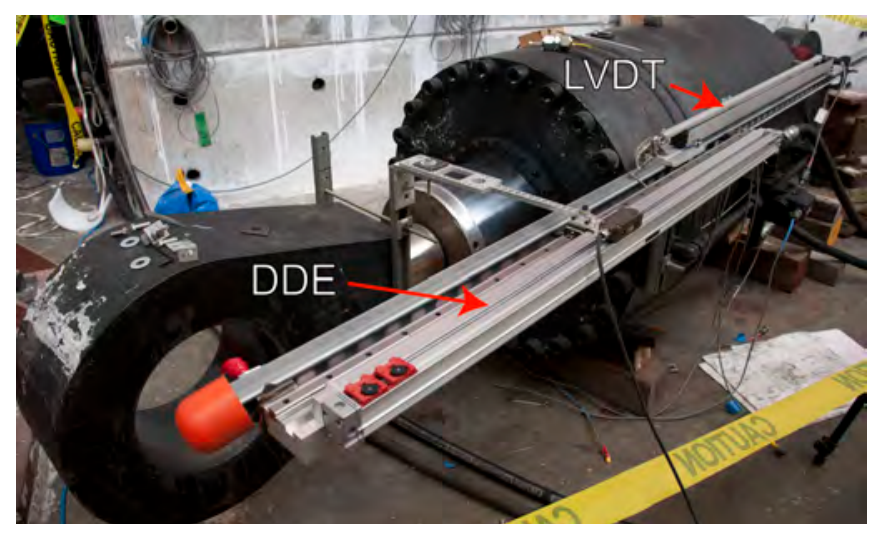

Figure 1: DDE verification using the servo-hydraulic actuator without a specimen.

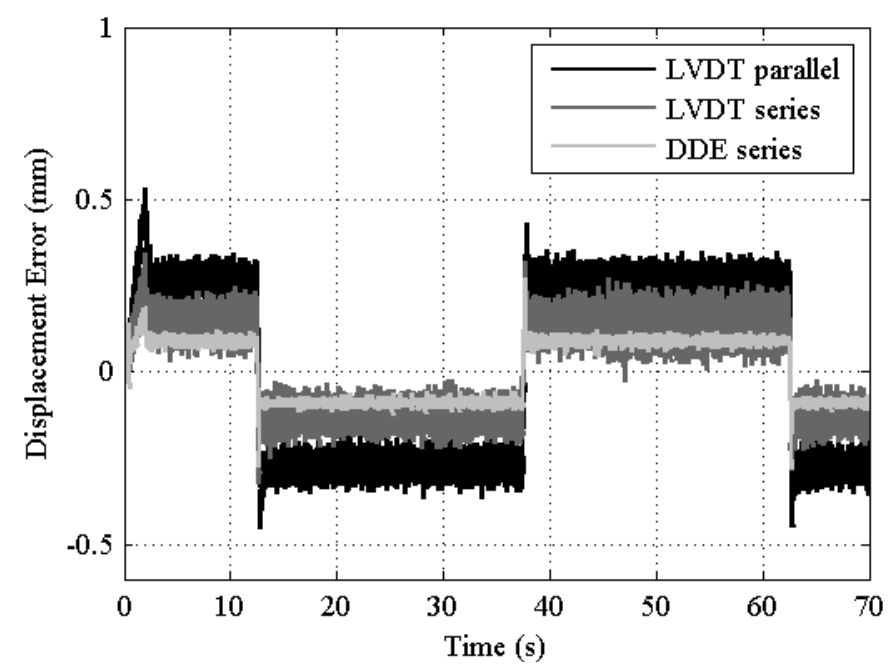

Figure 2: Displacement errors for LVDT parallel, LVDT series, and DDE series wiring using the unrestrained servo-hydraulic actuator at velocity $=2.032 \mathrm{~mm} / \mathrm{s}$.

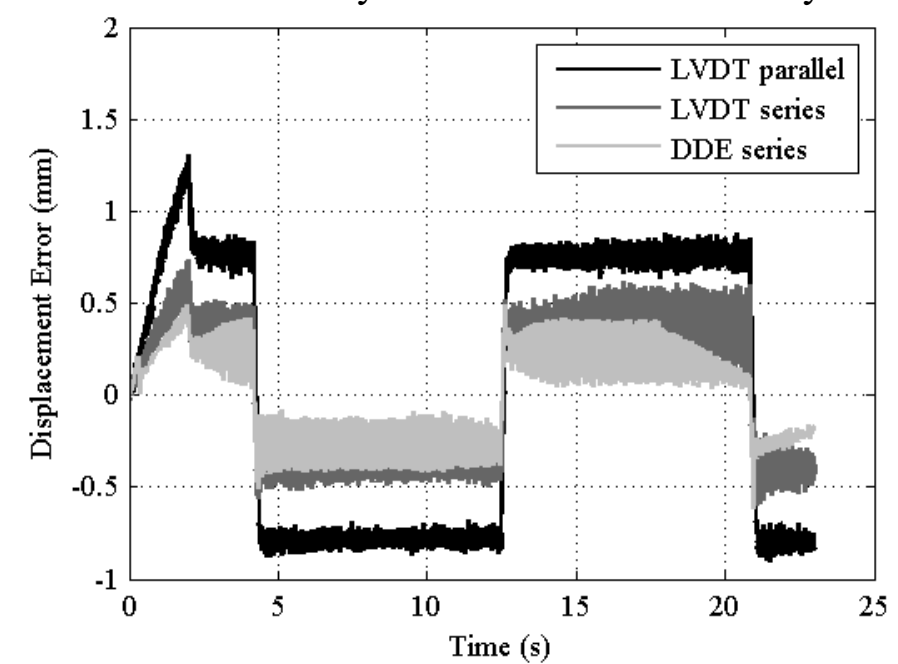

Figure 3: Displacement errors for LVDT parallel, LVDT series, and DDE series wiring using the unrestrained servo-hydraulic actuator at velocity $=6.096 \mathrm{~mm} / \mathrm{s}$. 


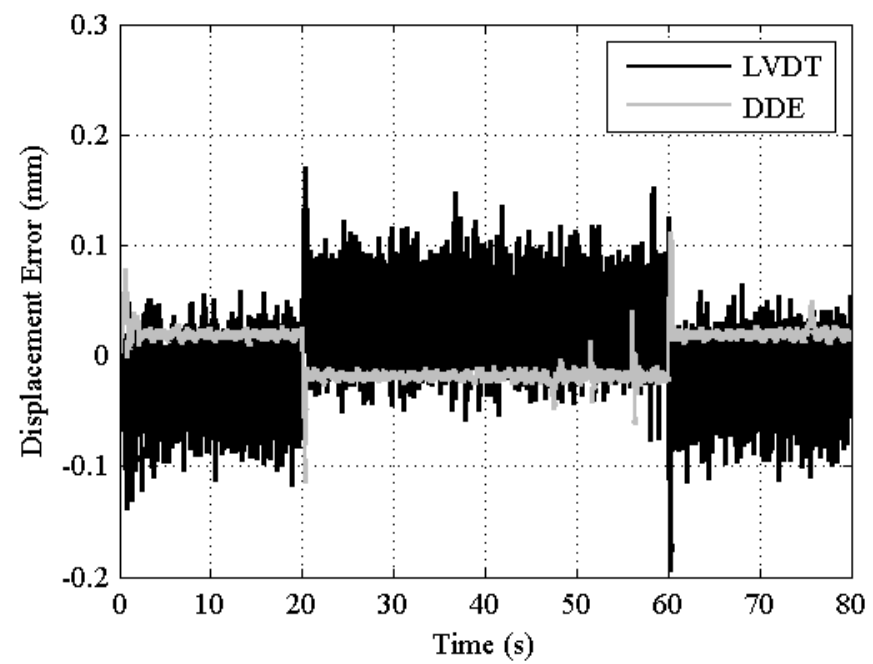

Figure 4: Displacement errors for LVDT and DDE servo-hydraulic actuator control using the braced frame specimen. 

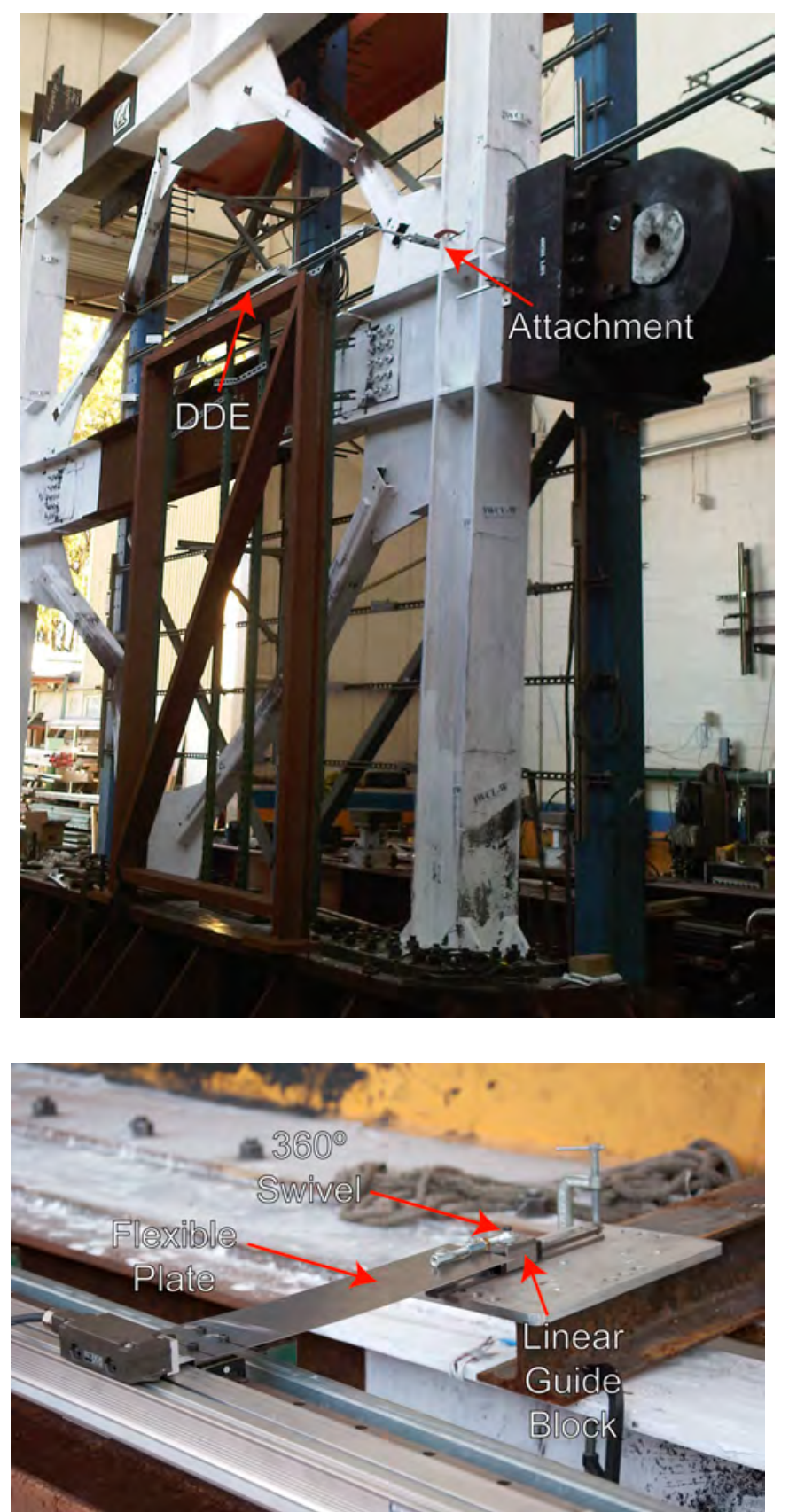

Figure 5a: Braced frame specimen, reused for DDE validation.

Figure 5b: DDE connection designed for hybrid simulations of the response of squat shear wall specimens; here, tested using the braced frame specimen. 


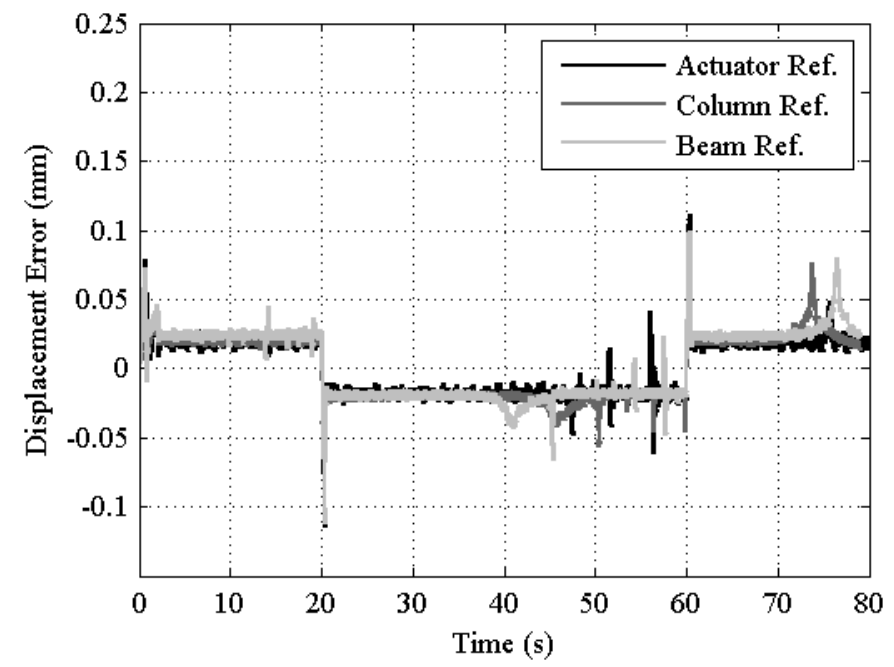

Figure 6: Displacement errors for DDE control using actuator, column, or beam reference points with the braced frame specimen.

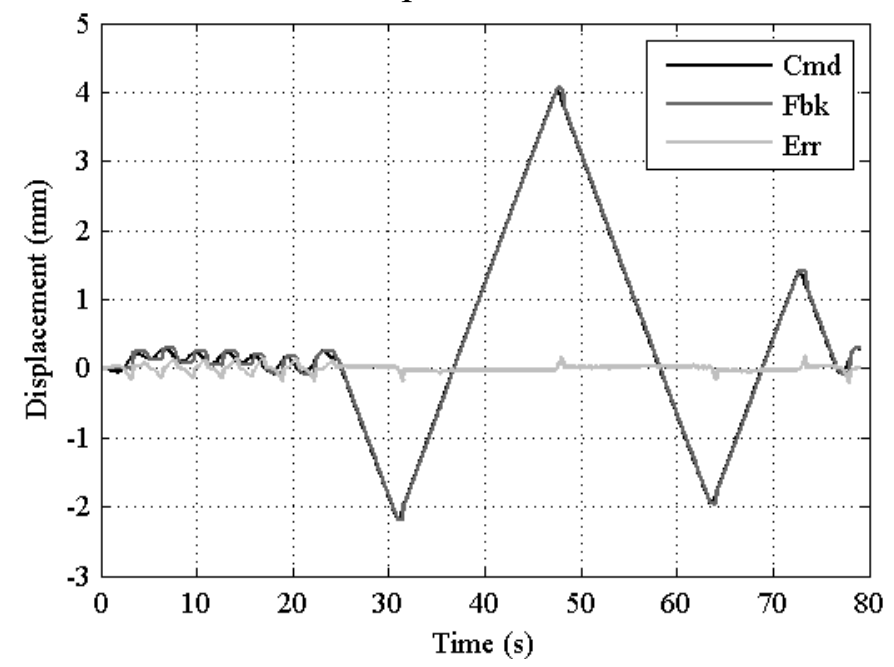

Figure 7: Displacement commands, feedbacks, and errors for hybrid simulation of the response of the braced frame specimen to $10 \%$ of the 1978 Tabas Earthquake ground motion. 


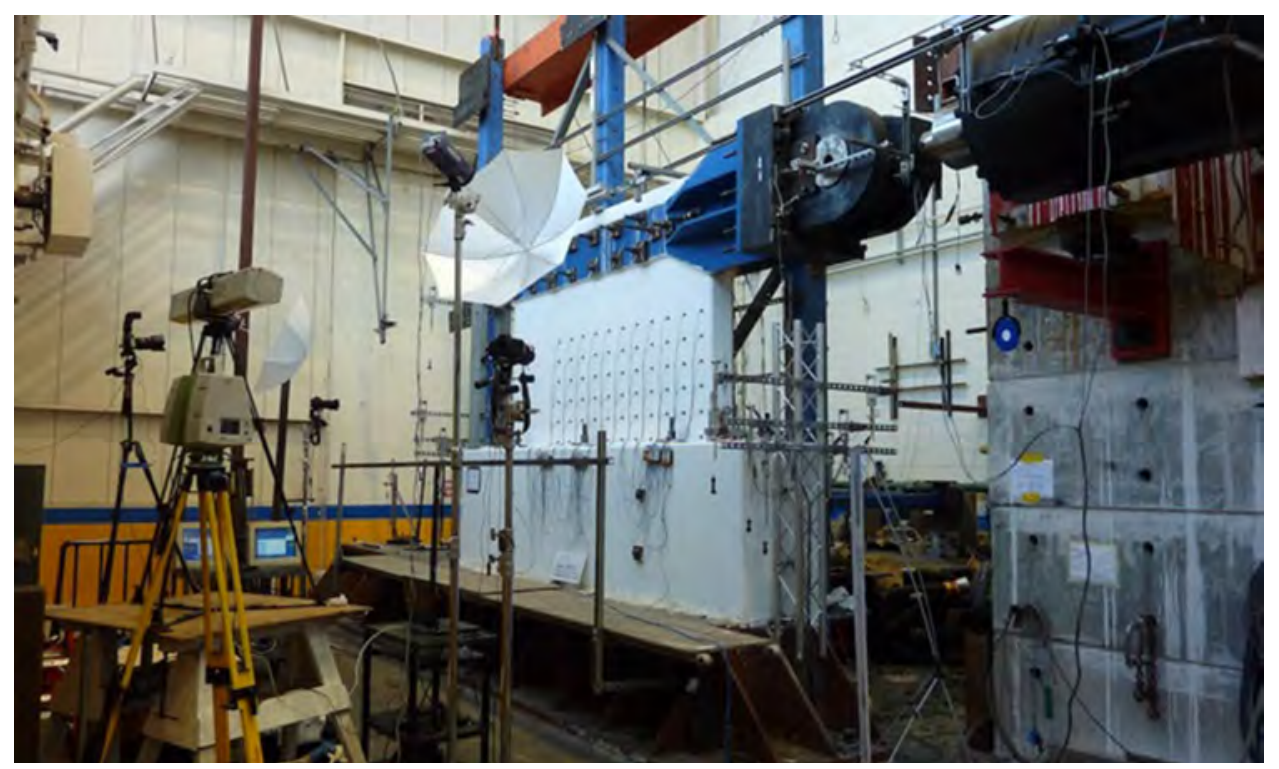

Figure 8: Global view of the squat reinforced concrete shear wall specimen test setup.
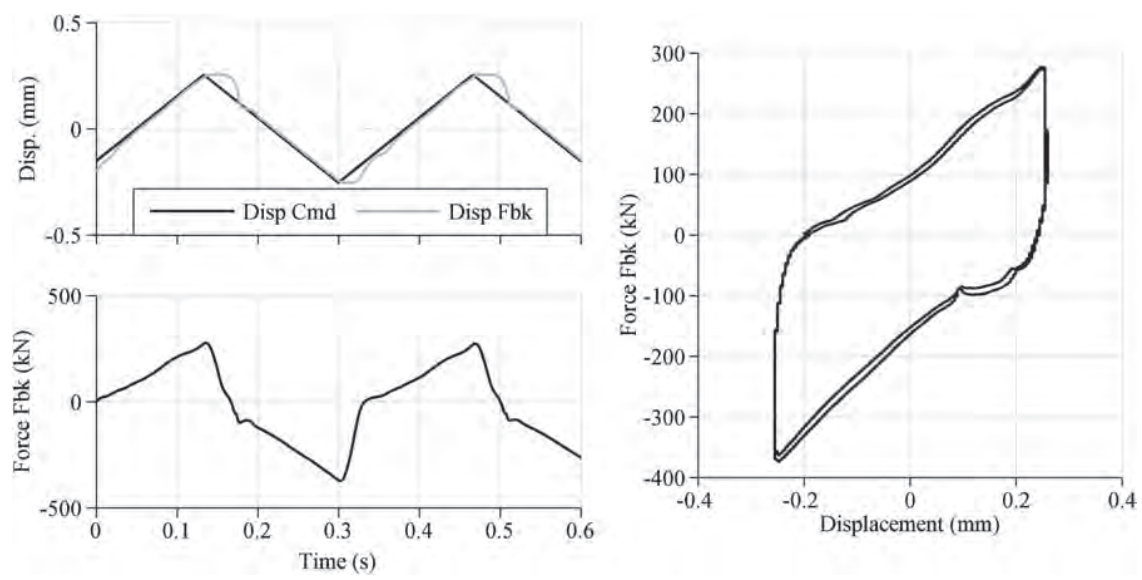

Figure 9: Wall 1 stiffness test results.
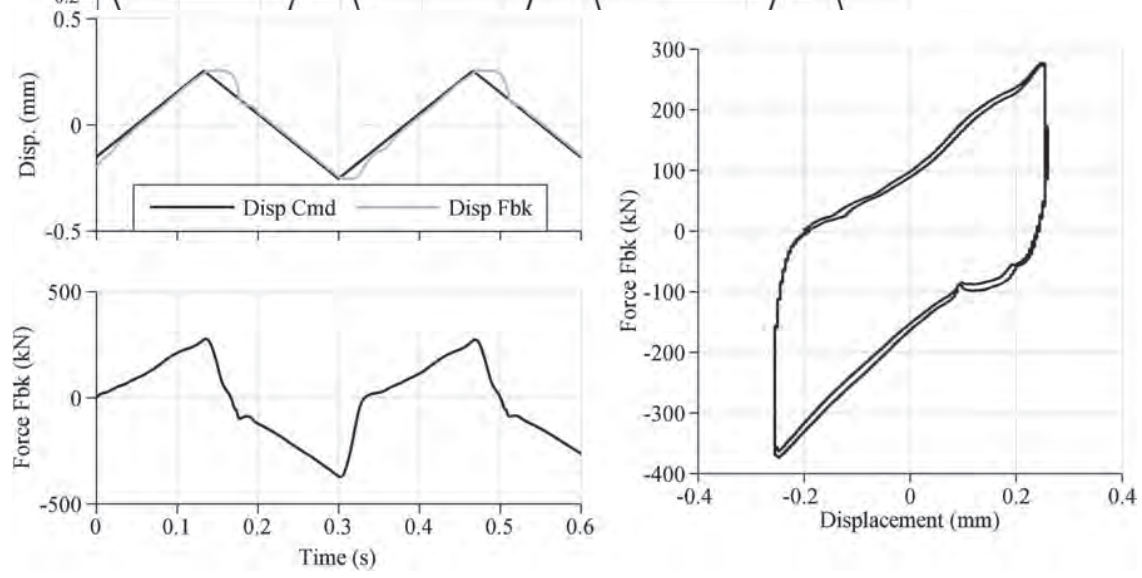

Figure 10: Wall 1 free vibration test results. 

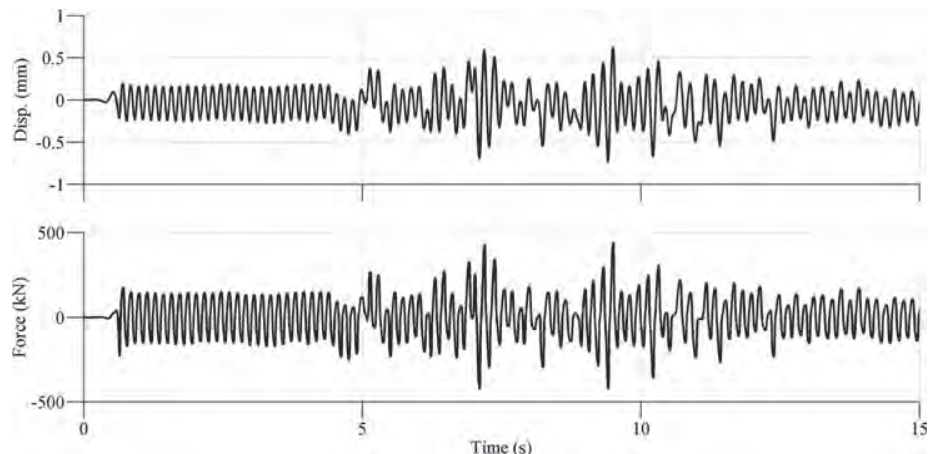

Figure 11: Wall 1 OBE displacement and force time histories. 